\section{$\underset{\substack{\text { hommes } \\ \text { \& migrations }}}{ }$}

\section{Hommes \& migrations}

Revue française de référence sur les dynamiques

migratoires

\section{$1284 \mid 2010$}

Migrations et environnement

Bangladesh, un pays fait de catastrophes

\title{
Bangladesh, un pays fait de catastrophes
}

La vulnérabilité environnementale et la migration forcée

\section{Alice Poncelet}

\section{(2) OpenEdition \\ Journals}

\section{Édition électronique}

URL : http://journals.openedition.org/hommesmigrations/1235

DOI : 10.4000/hommesmigrations. 1235

ISSN : 2262-3353

Éditeur

Musée national de l'histoire de l'immigration

Édition imprimée

Date de publication : 1 mars 2010

Pagination : 16-27

ISSN : 1142-852X

Référence électronique

Alice Poncelet, « Bangladesh, un pays fait de catastrophes », Hommes \& migrations [En ligne], 1284 I

2010, mis en ligne le 29 mai 2013, consulté le 10 décembre 2020. URL : http://

journals.openedition.org/hommesmigrations/1235; DOI : https://doi.org/10.4000/

hommesmigrations.1235 


\section{Bangladesh, \\ un pays fait de catastrophes La vulnérabilité environnementale et la migration forcée}

Par Alice Poncelet, Centre d'études de l'ethnicité et des migrations (Cedem), Université de Liège

Le Bangladesh, en raison de ses caractéristiques géophysiques, hydrologiques et de l'exploitation de ses ressources, fait partie des pays les plus vulnérables aux changements du climat. II est traversé par sept grands fleuves et plus de $\mathbf{2 0 0}$ cours d'eau qui définissent ainsi la géographie du pays et le style de vie de son peuple. Les populations établies sur les bords des cours d'eau sont régulièrement soumises aux inondations, cyclones et autres sécheresses. L'érosion des rives qui menace leur habitat rend leur existence précaire. Face aux aléas du climat, les Bangladeshis n'ont pas d'autre choix que la migration. 
L'Asie du Sud est considérée, par la majeure partie de la communauté scientifique, comme l'une des régions du monde les plus affectées par les changements climatiques. Selon le quatrième rapport d'évaluation de l'IPCC en 2007, les principaux impacts des changements climatiques dans la région sont : une augmentation des fréquences de sécheresses et d'inondations affectant la production locale ; l'élévation du niveau de la mer exposant les côtes à des risques de disparition et de destruction de plus en plus accrus ; l'érosion des terres tant en zone côtière que fluviale ; l'accélération de la fonte des glaciers de l'Himalaya, donc des crues et des coulées de boue détruisant ces zones. Certains pronostics indiquent que les rendements agricoles pourraient diminuer jusqu’à 30 \% en Europe centrale et en Asie du Sud pour le milieu du XXI' siècle $^{(1)}$.

La superficie totale du Bangladesh - seulement $144000 \mathrm{~km}^{2}$ pour une population d'environ 150 millions d'habitants - en fait l'un des pays les plus densément peuplés du monde (1 050 habitants au $\mathrm{km}^{2}$ ). Le Bangladesh étant un delta, la plupart de ses régions se situent à moins de douze mètres au-dessus du niveau de la mer. Il est prévu qu'environ 50 \% des terres soient inondées si le niveau de la mer augmente ne fût-ce que d'un mètre.

Ainsi, les désastres environnementaux sont des phénomènes récurrents dans ce pays. Linondation des terres agricoles en période de mousson conduit à des pertes de récoltes, de propriétés et de vies humaines assez conséquentes. L'érosion des berges causée par le déplacement continu des sillons dans les principaux fleuves - le Jamuna (Brahmapoutre en Inde), le Padma (le Gange) et le Meghna - est un problème endémique qui déplace entre 500000 personnes chaque année, selon le Bureau de gestion des catastrophes (Disaster Management Bureau, DMB) et 1 million, selon Islam $^{(2)}$. Ce déplacement de population régulier crée beaucoup de détresse dans le pays. L'érosion des berges est considérée comme l'un des principaux facteurs de paupérisation et de marginalisation des familles rurales, en raison de la perte de terres agricoles productives. Si bien que pour vivre dans ces zones fluviales, la population met sur pied des stratégies plus correctives que préventives. Ces stratégies correctives dépendent de leurs faibles possibilités technologiques et de leur absence de droits et statuts socio-économiques. Par conséquent, les inondations occupent une position unique dans l'économie et la culture du Bangladesh. Les habitants ont l'habitude de vivre avec les crues régulières qu'ils savent appréhender. Néanmoins, leurs moyens de subsistance sont de plus en plus menacés en raison de l'impact croissant du changement climatique.

Le concept de "déplacé climatique" étant encore à ses débuts sur la scène scientifique internationale, cette étude ${ }^{(3)}$ s'attelle à démontrer l'hypothèse selon laquelle les dernières vagues d'“exode rural" sont directement ou indirectement liées à la question des changements climatiques. 


\section{La disparition de la terre et l'invasion de l'eau salée}

Environ $80 \%$ de la population du Bangladesh vit dans les zones où seulement $40 \%$ possèdent des terres cultivables. L'érosion provoquant une destruction massive des terres, les habitants sont forcés de quitter leur lieu de résidence ${ }^{(4)}$ Environ 94 upazilas $^{(5)}$ et 50 districts doivent faire face à l'érosion des berges qui touche chaque année un nombre important de familles devenues "sans-abri". Les trois principaux fleuves du pays cités ci-dessus, ainsi que 16 autres rivières, sont les principaux lits des érosions.

Au moment de la mousson, un tiers du territoire est inondé. La population résidente a appris à s'adapter à ce problème climatique, mais le réchauffement global de la planète semble avoir brisé ce modèle. D'une part, l'augmentation des précipitations lors de la mousson, associée à la fonte de plus en plus importante des glaciers de l'Himalaya, a augmenté considérablement la quantité d'eau de crue. D'autre part, l'élévation du niveau de la mer rend l'écoulement de plus en plus difficile. Les inondations annuelles devraient gagner progressivement en volume et en durée.

Les conséquences de l'érosion des berges et des inondations inhabituelles sur l'économie locale sont déterminantes, puisqu'à la fois elles réduisent fortement la quantité des terres agricoles cultivables et cultivées et, parallèlement, elles détruisent les infrastructures et les systèmes de communication. Ces impacts néfastes conduisent à l'appauvrissement de la population. Le Bangladesh est ainsi limité par ses ressources internes disponibles pour faire face aux risques de catastrophes environnementales. Ces catastrophes créent, de plus, de graves problèmes de non-emploi dans les zones rurales, d'innombrables bidonvilles urbains et aggravent ainsi la situation socioéconomique des personnes déplacées. L'extrême pauvreté des paysans devenus "sans terre" force une part importante de la population bangladeshie à s'installer sur des îles fluviales mouvantes appelées "chars" au Bangladesh.

Plus de cinq millions de personnes habitent ces îles sur les trois principaux fleuves. Constituées de sable et de limon, et remaniées par les courants lors de chaque mousson, ces îles sont toutes aussi fertiles que précaires. À l'apogée de la saison pluvieuse, il faut parfois seulement quelques jours pour dissoudre un char de plusieurs centaines d'hectares. La fonte des glaciers de l'Himalaya, amplifiée par le réchauffement climatique, en plus d'accroitre les inondations dans la région deltaïque, accentue de manière significative l'érosion des terres le long des berges. Lorsqu'elle arrive dans les lits de rivières non adaptés à ce nouvel afflux d'eau qui heurte la berge très violemment, elle détruit et emporte sur son passage les cultures, les maisons et engloutit des hectares de terre. Les paysans des chars craignent cette 
érosion qui crée de nombreux dommages, même si elle ne cause que très rarement des pertes humaines. "Il arrive souvent de s'endormir riche propriétaire terrien et de se réveiller sans-abri", souligne un habitant de char. Bien que le phénomène existe depuis longtemps, sa fréquence a considérablement augmenté ces dernières années.

Plus de 5 millions de Bangladeshis vivent dans ces zones côtières très vulnérables. Le pays a connu 48 cyclones majeurs entre 1584 et 2007. Selon la plupart des estimations, l'intensité et la fréquence des cyclones sont susceptibles d'augmenter ${ }^{(6)}$. Avec deux événements météorologiques extrêmes, l'année 2007 a été unique dans l'histoire des catastrophes du Bangladesh (inondations inégalées de juillet à août et cyclone Sidr). Alors que la réhabilitation suite aux inondations était en cours, la partie côtière du pays a été de nouveau frappée par le cyclone Sidr d'une vitesse de $240 \mathrm{~km} / \mathrm{h}$. Il a touché 30 districts (sur 64), a très fortement endommagé les maisons, les terres agricoles et a conduit à la perte de vies humaines et d'animaux.

En plus de ces destructions, les inondations et cyclones augmentent la salinité de l'eau, entraînant une pénurie d'eau douce et menaçant l'écosystème qui en dépend. L'augmentation d'un mètre du niveau de la mer pourrait entraîner une perte de $20,7 \%$ des terres qui concernerait 14,8 millions de personnes. 


\section{Migrer sous la menace des éléments}

La plupart des experts s'accordent à dire que les flux migratoires au Bangladesh sont principalement des mouvements internes des zones rurales vers les zones urbaines. La migration se produit principalement pour des raisons économiques, sociales et environnementales. Ces facteurs ne fonctionnent pas de manière indépendante et séparée. Au contraire, ils se cumulent généralement et se renforcent mutuellement. Dans le cas du Bangladesh, il y a une distinction à faire entre les problématiques du Nord et celles rencontrées au Sud. La population du Nord développe des stratégies afin de faire face aux changements et aux lentes évolutions de l'environnement tels que l'accroissement de l'érosion, des périodes de mongas $^{(7)}$. Dans les régions côtières, vu le caractère parfois imprévisible et instantané des catastrophes, les décisions sont parfois très peu réfléchies et, par conséquent, les trajectoires migratoires dans cette région sont beaucoup moins organisées et plus soudaines. La plupart des réfugiés climatiques rencontrés ont été interrogés dans les bidonvilles de Dhaka (Tejgaon, Kawranbazar, Bangla Motor Uttora, Banani, Mohakhali, Farmegate et Mohammadpur). Le choix de la destination des migrants dépend à la fois de leur lieu de résidence d'origine, de leur activité économique, de leur réseau social, mais aussi de la période durant laquelle ils décident de migrer.

Après une catastrophe, les ménages bangladeshis déploient différentes stratégies pour développer leurs moyens de subsistance. Dans certains cas, le père émigre temporairement vers les centres urbains, tels que la capitale,Dhaka, mais aussi vers 
des grandes villes plus proches du lieu d'origine, comme Khulna ou Barisal, dans le but de travailler et d'envoyer de l'argent à la famille. D'autres migrent de manière saisonnière en suivant le marché du travail. Certains ont pris la décision de migrer avec la totalité de la famille pendant que d'autres ont choisi de ne pas émigrer, car le processus est complexe et demande un minimum de ressources. Cet investissement financier n'est pas envisageable pour les familles les plus pauvres.

La migration, qu'elle soit vers Dhaka ou vers l'Inde, est un choix forcé pour tous les migrants interrogés. Aucun d'entre eux ne désirait a priori migrer. La vie à Dhaka est très difficile et moins satisfaisante que prévu. Les nouveaux migrants luttent pour trouver un emploi et deviennent des travailleurs journaliers, changeant de "métiers" tous les jours en fonction de l'offre (casseur de briques, chauffeur de rickshaw, ouvrier dans l'industrie du textile).

Contrairement au discours officiel du Département de gestion de l'alimentation et des catastrophes, les gens sont trop pauvres

\section{Les périodes de sécheresse qui durent plus longtemps et l'intensification de la fréquence des catastrophes naturelles accentuent la pauvreté des sols et la médiocre qualité de l’eau.} pour migrer en fonction des seules raisons sociales et éducatives. De nombreux bidonvilles de migrants ont été détruits par le gouvernement. Ce dernier n'a prévu aucun plan de réhabilitation ou de réinstallation de la population ${ }^{(8)}$. Face à une telle situation, les gens sont obligés de se déplacer d'un bidonville à l'autre. Cela accentue leur précarité et les pousse à rejoindre leur lieu de résidence d'origine.

Les migrations temporaires sont la forme la plus fréquente de migrations liées à des catastrophes naturelles. Pendant la saison des crues, la migration tend à être saisonnière. En opposition, les travailleurs qui ont perdu leur emploi dans la catastrophe, comme ceux qui ont perdu leurs biens, ont tendance à chercher à s'installer de manière permanente, car ils ne trouvent plus de raisons de se battre dans un milieu hostile où ils n'ont plus rien à sauver.

Les principaux facteurs énoncés, qui semblent influencer la décision de migrer des acteurs, sont les problèmes de non-emploi, d'insuffisance de revenus, de terres à cultiver et de moyens de subsistance, et les mauvaises conditions de vie. Tous ces facteurs sont, entre autres, les conséquences des dégradations de l'environnement. Les périodes de sécheresse qui durent plus longtemps et l'intensification de la fréquence des catastrophes naturelles accentuent la pauvreté des sols et la médiocre qualité de l'eau. Toutes ces raisons contribuent à la paupérisation de la population et par conséquent à leur décision de migrer. 
Dans leur majorité, ces migrants aimeraient réinvestir leur résidence d'origine si la situation environnementale s'améliorait. En migrant, beaucoup ont quitté leurs proches et voudraient rentrer vivre auprès d'eux. Au Bangladesh, les réseaux familiaux sont forts et le restent, malgré la migration et les difficultés de communication.

\section{Le portrait de ceux qui restent}

En réalité, la plupart des personnes qui choisissent de rester ont effectivement une histoire de migration, cependant, ils n'ont pas (encore) pris la décision de migrer vers une zone définitivement plus sûre. Leurs stratégies sont analysées séparément des personnes identifiées et définies comme étant des migrants.

Dans le Nord, de la frontière indienne aux chars du district de Gaibandha, la plupart des non-migrants ont une histoire migratoire assez importante, mais leurs déplacements se restreignent à un périmètre très limité au sein de leur région d'origine, c'est-à-dire d'un char à l'autre et cela parfois jusqu'à dix fois par an. Ces personnes ne se considèrent pas comme étant des migrants. Ils élaborent des stratégies d'adaptation pour faire face aux inondations et à l'érosion des berges. Ils savent, cependant, que si la situation perdure et s'aggrave ils devront trouver une solution à plus long terme et migrer. En outre, de nombreuses familles pauvres ne peuvent pas se permettre de La population vivant dans
les chars est parmi la plus
pauvre au Bangladesh.
Ce va-et-vient de terres
habitables et cultivables
induit des conflits de
régimes fonciers, aggravant
ainsi l'appauvrissement
de la population. se déplacer sur une plus grande distance, à cause du manque de ressources et des incertitudes qu'implique ce type de migration. Enfin, les personnes issues des chars sont un groupe distinct avec une culture distincte.

Dans la majorité des situations, les gens n'ont pas de titre de propriété, ce qui les empêche d'abandonner leurs terres. Car la bataille pour la terre est ardue. Le premier arrivé sur la terre réémergente est celui qui pourra l'exploiter. La population vivant dans les chars est parmi la plus pauvre au Bangladesh. Ce va-etvient de terres habitables et cultivables induit des conflits de régimes fonciers, aggravant ainsi l'appauvrissement de la population. Les gens perdent leur titre de propriété au moment où ils perdent leurs terres sous l'eau et deviennent ainsi "locataires" sur de nouvelles terres. 
À l'instar des personnes interviewées dans le nord du Bangladesh, peu de Bangladeshis vivant dans le Sud ne se sont jamais déplacés. Tous ont, à un moment donné, dû mettre en place des mécanismes pour survivre aux changements climatiques et économiques. Ces stratégies d'adaptation et ces décisions de migrer ne sont pas facilement prises par la population locale. Elles sont fonction de périodes et de raisons différentes.

Ainsi, plusieurs types de nonmigrants dans le Sud sont identifiés. Il y a ceux qui trouvent encore des stratégies d'adaptation et qui s'en satisfont. Ce sont majoritairement les personnes qui ont réussi à trouver un emploi dans les cultures de crevettes, dans la récolte de sel ou dans tout autre domaine d'activités qui subsistent après les catastrophes. Cependant, il est clair que ces personnes ne sont pas une majorité puisque ces adaptations demandent moins de main-d'ceuvre.

Il y a des migrants qui amorcent leur processus migratoire en faisant une migration de proximité. Ce sont des personnes qui viennent de l'extrême sud et des villages complètement dévastés, qui remontent petit à petit vers Dhaka, mais qui préfèrent dans un premier temps s'arrêter et essayer de se réinstaller dans le centre urbain le plus proche de leur village d'origine.

Ces non-migrants sont, par ailleurs, soit des personnes trop pauvres pour migrer, soit des personnes qui ont des biens propres et qui ne sont pas encore prêtes à s'en séparer. Malgré le fait d'habiter toujours dans des zones très dangereuses, elles préferrent ne pas migrer et conserver jalousement leur lopin de terre à cultiver, un semblant de commerce ou quelques bêtes d'élevage.

Enfin, ils peuvent avoir essayé de migrer en Inde mais en sont revenus, car ils étaient victimes de réseaux de trafiquants d'êtres humains. 


\section{Les liens entre la dégradation de l'environnement et la migration}

Bien que la dégradation de l'environnement en tant que telle ne soit pas encore reconnue comme un des principaux moteurs de la migration au Bangladesh, on lui reconnaît des interdépendances avec d'autres facteurs de migration. Les catastrophes environnementales ont des conséquences sur la situation de l'emploi, tant dans les lieux d'origine que dans les zones urbaines où s'installent les migrants.

Dans le Nord, pendant la saison des pluies, des hectares de terres et de cultures sont détruits et inondés. Ces mêmes terres sont menacées de désertification durant la saison sèche. Tant pour des raisons géologiques que pour des raisons de propriété des sols, il est très difficile de vivre de l'agriculture, c'est pourquoi de nombreux hommes redirigent leur activité économique vers le monde de la pêche. Ils doivent dès lors faire face à des problèmes de surpêche ce qui les oblige soit à trouver une nouvelle activité, soit à migrer vers les grandes villes.

Dans le Sud, les changements environnementaux dus au climat ou aux constructions humaines en amont sur les fleuves (barrage, etc.) provoquent la salinisation de l'eau douce. Le processus est aggravé au cours de la saison cyclonique. Les rizières ne sont plus viables, et les agriculteurs doivent s'adapter. Les cultures de riz ont été remplacées par l'élevage de crevettes. Cependant cette activité ne demande pas beaucoup de main-d'cuvre, ce qui conduit à l'accroissement du chômage. De plus, les crevettes sont trop coûteuses pour être consommées localement, et la pénurie de l'approvisionnement en riz en augmente fortement le prix, ce qui conduit la population à l'insécurité alimentaire. 
Les activités économiques ont donc fortement évolué ces dernières années dans les régions sinistrées à cause des changements environnementaux et des migrations. Il n'est donc pas étonnant que dans des mégapoles comme Dhaka, les métiers et l'organisation économique évoluent aussi. C'est ainsi que "les métiers des migrants" ont vu le jour. De chauffeur de rickshaw, à casseur de briques ou porteur de sacs de ciment, chaque matin, c'est une nouvelle recherche d'emploi commence pour ces "travailleurs d'un jour" qui vivent de leur activité économique dans la précarité et l'instabilité des bidonvilles.

\section{Les femmes et la traite des êtres humains}

La plupart des stratégies d'adaptation adoptées par les ménages impliquent le départ des hommes (ou leur décès). Cela suppose que les femmes sont laissées seules avec leurs enfants dans les villages. Souvent plus pauvres, elles ont moins accès aux ressources, ce qui diminue leur capacité à s'adapter aux changements environnementaux. Les trafiquants et passeurs vers la frontière indienne voisine profitent de cette situation. Ils promettent à ces femmes ou à ces ménages affaiblis de les aider à passer en Inde afin qu'ils se réinstallent dans des villes plus sécurisantes où la possibilité de trouver du travail et d'avoir un revenu est bien réelle. Hélas, ces passeurs sont trop souvent des escrocs qui profitent de la vulnérabilité de leurs victimes pour s'enrichir et les vendre comme "esclaves" sexuelles ou "esclaves" dans d'importantes manufactures de confection de textile situées à la frontière indienne. Plusieurs types de trafic ont été observés.

Le premier type et le plus fréquent concerne les femmes (veuves ou vivant seules) et leurs enfants. Rendues vulnérables économiquement et physiquement par l'absence de leur mari, elles deviennent des proies faciles pour les trafiquants. Elles se retrouvent dans des réseaux de prostitution ou de travail forcé.

Le second type de victimes concerne les hommes. Après les périodes d'inondations, ils sont approchés par les passeurs dans le but de franchir la frontière indienne dans l'espoir de trouver du travail et une vie plus stable. Les passeurs-escrocs les envoient en réalité dans des ateliers clandestins où ils sont traités en esclaves.

Pour le dernier type, le recours aux passeurs et à la traite humaine est considéré comme une stratégie d'adaptation. Certaines familles décident de vendre leurs filles à des escrocs et proxénètes en toute conscience et en connaissance de cause. Ces trafics ne sont pas nouveaux au Bangladesh, cependant ils ont été renforcés par les désastres environnementaux. 


\section{Conclusion}

Les désastres provoqués par les inondations et l'érosion sont des phénomènes complexes dans le contexte actuel du Bangladesh. Bien que des incertitudes demeurent sur l'ampleur du changement climatique, il est un fait que des millions de personnes ont récemment été touchées par les inondations et la pénurie alimentaire saisonnière, nécessitant l'évacuation de la population, la mise en place d'abris et d'un système de secours efficace.

La prise de conscience par la population bangladeshie que ces catastrophes et leurs conséquences ne sont pas uniquement un hasard naturel reste très incertaine. D'importants mouvements de population sont clairement liés à ces catastrophes environnementales (érosion, cyclones, eau salée, etc.) et les changements climatiques vont considérablement aggraver cette situation.

Les mouvements actuels de la population ne sont ni contrôlés ni gérés. D'un même désastre environnemental peuvent déboucher différentes stratégies d'adaptation et différents modèles migratoires. À l'heure actuelle, au Bangladesh, la migration (temporaire ou permanente) apparaît comme l'une des meilleures stratégies pour les populations touchées. La migration est cependant très difficile et entravée par de nombreux obstacles. Elle n'est pas encouragée ni soutenue par des politiques adéquates ou des structures de soins pour les migrants touchés par une grande précarité.

La population tente actuellement de parer aux conséquences immédiates des catastrophes, mais il viendra un temps où l'adaptation locale ne sera plus possible. Le Bangladesh pourrait alors devenir un théâtre de vastes mouvements de populations déclenchés par le changement climatique, ce qui pourrait constituer une menace pour la sécurité régionale.

Pour appréhender cette problématique, Zaman insiste sur la nécessité d'une approche holistique des problèmes d'inondation et d'érosion qui reconnaitrait les interactions complexes avec l'organisation de la société bangladeshie ${ }^{(9)}$. Islam ${ }^{(10)}$ permet d'amorcer cette vision holistique et systémique de la problématique des catastrophes environnementales dues aux changements climatiques en concevant l'environnement comme un système d'interdépendances entre l'environnement physique et social. 
Bibliographie

- C. R. Abrar and S. N. Azad, Coping with Displacement. Riverbank Erosion in North-West Bangladesh, Dhaka, North Bengal Institute, RDRS and RMMRU, 2004.

- A. Ali, "Vulnerability of Bangladesh to Climate Change and Sea Level Rise Through Tropical Cyclones and Storm Surges", in Water, Air, \& Soil Pollution, n²2, 1996, pp. 171-179.

- Association for community development (ACD), "River Bank Erosion and Its Impact on Displaced People in Two Districts of Bangladesh", unpublished working document collected during fieldwork in Bangladesh.

- Community-Based Disaster Management (CBDM) in South Asia, Bangladesh Case Study, vol. 1, n¹, 2008. http://www.adpc.net/v2007/IKM/ONLINE\%2ODOCUMENTS/downloads/2008/1_CaseStudyCBDMStructureFinal.pdf

- The Women's Environment and Development Organization(WEDO), Gender, Climate Change and Human Security, Lessons from Bangladesh, Ghana and Senegal, report prepared for ELIAMEP, 2008.

- M. M. Hossain, "Shifting Characteristics of the Ganges in Bangladesh", in K. M. Elahi, K. S. Ahmed and M. Mafizuddin (éds.), River Bank Erosion Impact Study, Savar, Jahangirnagar University, 1991.

- IPCC (Intergovernmental Panel on Climate Change), Summary for Policymakers. Climate Change 2007: Impacts, Adaptation and Vulnerability, Contribution of Working Group II to the Fourth Assessment Report of the IPCC, 2007, pp. 7-22.

- M. Zulfiquar Ali Islam, Indigenous Adaptation Strategies of the Riverbank Erosion Displacees in Bangladesh: A Study of Two Northwestern Riparian Villages, paper presented at the International Human Dimensions Programme on Global Environmental Change (IHPD), Open Meeting, 2009.

- Alice Poncelet, Bangladesh Case Study Report for the Environmental Change and Forced Migration Scenarios (Each-For) Project, Brussels, European Commission, Sixth Framework Programme (FP6), 2009. www.each-for.eu

- M. Zaman, "Flood and Erosion Disasters in Bangladesh: the Human Dimensions", Vancouver, document non publié collecté durant l'étude de terrain au Bangladesh, 2007.

\section{Notes}

1. ELIAMEP, Gender, Climate Change and Human Security, Lessons from Bangladesh, Ghana and Senegal, May 2008.

2. La récolte de données et de chiffres fiables au Bangladesh n'est pas chose aisée, d'autant plus qu'il n'existe pas de recensement systématique de la population. Nous voulons dès lors insister sur la prudence à avoir lors de la lecture de ces chiffres dans cet article, puisqu'ils ne sont qu'une vague estimation et non des données précises.

3. Cet article est basé sur un terrain de recherche mené au printemps 2008 dans le cadre du projet EACH-FOR. La recherche s'est fondée sur des entretiens d'experts menés principalement dans la capitale Dhaka, dans les régions de Gaibandha et de Sathkira, et dans le district de Khulna, ainsi que sur des entretiens semi-structurés accompagnés de questionnaires standardisés directement réalisés avec les migrants dans les bidonvilles de Dhaka et les personnes touchées dans le sud-ouest et dans le nord-ouest du pays.

4. M. M. Hossain, "Shifting Characteristics of the Ganges in Bangladesh", in K. M. Elahi, K. S. Ahmed and M. Mafizuddin (éds.), River Bank Erosion Impact Study, Savar, Jahangirnagar University, 1991, p. 307.

5. Le Bangladesh est composé de six régions administratives appelées Bibhag divisées en 64 districts (zilas) sous-divisés en upazilas, plus petit niveau administratif au Bangladesh.

6. Intergovernmental Panel on Climate Change, Summary for Policymakers. Climate Change 2007:Impacts, Adaptation and Vulnerability, Contribution of Working Group II to the Fourth Assessment Report of the IPCC, 2007, pp. 7-22.

7. Période de pénurie alimentaire saisonnière

8. Rappelons que la situation décrite correspond à la réalité étudiée en mars 2008.

9. M. Zaman, "Flood and Erosion Disasters in Bangladesh: the Human Dimensions", Vancouver, document non publié collecté durant l'étude de terrain au Bangladesh, 2007.

10. M. Zulfiquar Ali Islam, Indigenous Adaptation Strategies of the Riverbank Erosion Displacees in Bangladesh: A Study of Two Northwestern Riparian Villages, paper presented at the International Human Dimensions Programme on Global Environmental Change (IHPD), Open Meeting, 2009. 